\title{
Enhancing The Performance Of A Short Run Multivariate Control Chart For The Process Mean
}

\author{
Michael B. C. Khoo \\ UniversitiSains, Malaysia, mkbc@usm.my \\ T. F. Ng \\ Universiti Sains, Malaysia
}

Follow this and additional works at: http://digitalcommons.wayne.edu/jmasm

Part of the Applied Statistics Commons, Social and Behavioral Sciences Commons, and the Statistical Theory Commons

\section{Recommended Citation}

Khoo, Michael B. C. and Ng, T. F. (2005) "Enhancing The Performance Of A Short Run Multivariate Control Chart For The Process Mean," Journal of Modern Applied Statistical Methods: Vol. 4 : Iss. 1 , Article 21.

DOI: $10.22237 /$ jmasm/1114906860

Available at: http://digitalcommons.wayne.edu/jmasm/vol4/iss1/21 


\title{
Enhancing The Performance Of A Short Run Multivariate Control Chart For The Process Mean
}

\author{
Michael B.C. Khoo T. F. Ng \\ School of Mathematical Sciences \\ Universiti Sains, Malaysia
}

Short run production is becoming more important in manufacturing industries as a result of increased emphasis on just-in-time (JIT) techniques, job shop settings and synchronous manufacturing. Short run production or more commonly short run is characterized by an environment where the run of a process is short. To meet these new challenges and requirements, numerous univariate and multivariate control charts for short run have been proposed. In this article, an approach of improving the performance of a short run multivariate chart for individual measurements will be proposed. The new chart is based on a robust estimator of process dispersion.

Key words: Short run, process mean, process dispersion, quality characteristic, in-control, out-of-control

Introduction

Let $\boldsymbol{X}_{n}=\left(X_{n 1}, X_{n 2}, \ldots, X_{n p}\right)^{\prime}$ denotes the $p \times 1$ vector of quality characteristics made on a part. Assume that $\boldsymbol{X}_{n}, n=1,2, \ldots$, are independent and identically distributed (i.i.d.) multivariate normal, $N_{p}(\mu, \Sigma)$, observations where $X_{n j}$ is the observation on variable (quality characteristic) $j$ at time $n$. Define the estimated mean vector obtained from a sequence of $\boldsymbol{X}_{1}, \boldsymbol{X}_{2}, \ldots, \boldsymbol{X}_{n}$ random multivariate observations as $\quad \bar{X}_{n}=\left(\bar{X}_{1}, \bar{X}_{2}, \ldots, \bar{X}_{p}\right)^{\prime} \quad$ where $\bar{X}_{j}=\sum_{i=1}^{n} X_{i j} / n$ is the estimated mean for variable $j$ made from the first $n$ observations. Table 1 gives the additional notations that are required in the article.

Michael B. C. Khoo (Ph.D., University Science of Malaysia, 2001) is a lecturer at the University of Science of Malaysia. His research interests are statistical process control and reliability analysis. Email: mkbc@usm.my. T. F. Ng is a graduate student in the school of Mathematical Sciences, University Science of Malaysia.
The following four cases (see Khoo \& Quah, 2002) of $\mu$ and $\Sigma$ known and unknown give the standard normal $V$ statistics for the short run multivariate chart based on individual measurements: Because $V$ statistics follow a standard normal distribution, this feature makes it suitable for the limits of the chart to be based on the 1-of-1, 3-of-3, 4-of-5 and EWMA tests which will be discussed in the later section.

Case KK: $\mu=\mu_{0}, \Sigma=\Sigma_{0}$, both known

$$
T_{n}^{2}=\left(X_{n}-\mu_{0}\right)^{\prime} \Sigma_{0}^{-1}\left(X_{n}-\mu_{0}\right)
$$

and

$$
V_{n}=\Phi^{-1}\left\{H_{p}\left(T_{n}^{2}\right)\right\}, n=1,2, \ldots
$$

Case UK: $\mu$ unknown, $\Sigma=\Sigma_{0}$ known

$$
T_{n}^{2}=\left(\boldsymbol{X}_{n}-\overline{\boldsymbol{X}}_{n-1}\right)^{\prime} \Sigma_{0}^{-1}\left(\boldsymbol{X}_{n}-\overline{\boldsymbol{X}}_{n-1}\right)
$$

and

$$
V_{n}=\Phi^{-1}\left\{H_{p}\left[\left(\frac{n-1}{n}\right) T_{n}^{2}\right]\right\}, n=2,3, \ldots
$$

Case KU: $\mu=\mu_{0}$ known, $\Sigma$ unknown

where

$$
T_{n}^{2}=\left(X_{n}-\mu_{0}\right)^{\prime} S_{0, n-1}^{-1}\left(X_{n}-\mu_{0}\right)
$$

$$
\boldsymbol{S}_{0, n}=\frac{1}{n} \sum_{i=1}^{n}\left(\boldsymbol{X}_{i}-\mu_{0}\right)\left(\boldsymbol{X}_{i}-\mu_{0}\right)^{\prime}
$$


and

$$
\begin{gathered}
V_{n}=\Phi^{-1}\left\{F_{p, n-p}\left[\left(\frac{n-p}{p(n-1)}\right) T_{n}^{2}\right]\right\}, \\
n=p+1, p+2, \ldots
\end{gathered}
$$

Case UU: $\mu$ and $\Sigma$ both unknown

$$
T_{n}^{2}=\left(\boldsymbol{X}_{n}-\overline{\boldsymbol{X}}_{n-1}\right)^{\prime} S_{n-1}^{-1}\left(\boldsymbol{X}_{n}-\overline{\boldsymbol{X}}_{n-1}\right)
$$

where

$$
S_{n}=\frac{1}{n-1} \sum_{i=1}^{n}\left(X_{i}-\bar{X}_{n}\right)\left(X_{i}-\bar{X}_{n}\right)^{\prime}
$$

and

$$
\begin{gathered}
V_{n}=\Phi^{-1}\left\{F_{p, n-p-1}\left[\left(\frac{(n-1)(n-p-1)}{n p(n-2)}\right) T_{n}^{2}\right]\right\}, \\
n=p+2, p+3, \ldots
\end{gathered}
$$

In Eq. (1) - (4), $p$ represents the number of quality characteristics that are monitored simultaneously, i.e., $p \geq 2$.

Enhanced Short Run Multivariate Control Chart for Individual Measurements

The short run multivariate chart statistics in Eq. (1) and (2) are based on the known covariance matrix while that of Eq. (3) and (4) are based on the estimated covariance matrix, a.k.a., the sample covariance matrix. It is shown in Ref. 1 that the performance of the chart based on the $V$ statistics in Eq. (3) and (4) are inferior to that of cases KK and UK in Eq. (1) and (2) respectively.

Thus, in this article an approach to enhance the performance of the short run multivariate chart for cases $\mathrm{KU}$ and $\mathrm{UU}$ is proposed by replacing the estimators of the process dispersion, i.e., $\boldsymbol{S}_{0, n}$ and $\boldsymbol{S}_{\boldsymbol{n}}$ in Eq. (3) and (4) respectively with a robust estimator of scale based on a modified mean square successive difference (MSSD) approach. Holmes and Mergen (1993) and Seber (1984) provided discussion about the MSSD approach. The new estimator of the process dispersion is denoted by $S_{\text {MssD }}$ while the new $V$ statistic is represented by $V_{\mathrm{MSSD}}$.

Table 1. Notations for Cumulative Distribution Functions.

$$
\begin{array}{|l}
\hline(.) \quad \text { - The standard normal cumulative distribution function } \\
\Phi^{-1}(.) \quad \begin{array}{l}
\text { - The inverse of the standard normal cumulative distribution } \\
\text { function }
\end{array} \\
H_{v}(.) \quad \begin{array}{c}
\text { - The chi-squared cumulative distribution function with } v \\
\text { degrees of freedom }
\end{array} \\
F_{v_{1}, v_{2}}(.) \text { - The Snedecor- } F \text { cumulative distribution function with }\left(v_{1}, v_{2}\right) \\
\text { degrees of freedom }
\end{array}
$$


The following formulas give the new standard normal $V_{\text {MSSD }}$ statistics for cases $\mathrm{KU}$ and UU: Note that all the notations which are used here are similar to that defined in the previous section.

Case KU: $\mu=\mu_{0}$ known, $\Sigma$ unknown

For odd numbered observations, i.e., $n$, is an odd number,

$$
T_{\mathrm{MSSD}, n}^{2}=\left(\boldsymbol{X}_{n}-\mu_{0}\right)^{\prime} S_{\mathrm{MSSD}, n-1}^{-1}\left(\boldsymbol{X}_{n}-\mu_{0}\right)
$$

where

$$
\boldsymbol{S}_{\mathrm{MSSD}, n-1}=\frac{1}{2} \sum_{i=2,4,6}^{n-1}\left(\boldsymbol{X}_{i}-\boldsymbol{X}_{i-1}\right)\left(\boldsymbol{X}_{i}-\boldsymbol{X}_{i-1}\right)^{\prime}
$$

and

$$
\begin{gathered}
V_{\mathrm{MSSD}, n}= \\
\Phi^{-1}\left\{F_{p, \frac{1}{2}(n-2 p+1)}\left[\frac{n-2 p+1}{2 p}\right] T_{\mathrm{MSSD}, n}^{2}\right\}, \quad n=2 p+
\end{gathered}
$$$$
1,2 p+3, \ldots
$$

For even numbered observations, i.e., $n$, is an even number,

$$
T_{\mathrm{MSSD}, n}^{2}=\left(\boldsymbol{X}_{n}-\mu_{0}\right)^{\prime} \boldsymbol{S}_{\mathrm{MSSD}, n-2}^{-1}\left(\boldsymbol{X}_{n}-\mu_{0}\right)
$$

where

$$
S_{\mathrm{MSSD}, n-2}=\frac{1}{2} \sum_{i=2,4,6}^{n-2}\left(\boldsymbol{X}_{i}-\boldsymbol{X}_{i-1}\right)\left(\boldsymbol{X}_{i}-\boldsymbol{X}_{i-1}\right)^{\prime}
$$

and

$$
\begin{aligned}
& V_{\mathrm{MSSD}, n}= \\
& \Phi^{-1}\left\{F_{p, \frac{1}{2}(n-2 p)}\left[\frac{n-2 p}{2 p}\right] T_{\mathrm{MSSD}, n}^{2}\right\}, \quad n=2 p+2,2 p \\
& +4, \ldots
\end{aligned}
$$

Case UU: $\mu$ and $\Sigma$ both unknown

For odd numbered observations, i.e., $n$, is an odd number,

$$
T_{\mathrm{MSSD}, n}^{2}=\left(X_{n}-\bar{X}_{n-1}\right)^{\prime} S_{\mathrm{MSSD}, n-1}^{-1}\left(X_{n}-\bar{X}_{n-1}\right)
$$

where

$$
\boldsymbol{S}_{\mathrm{MSSD}, n-1}=\frac{1}{2} \sum_{i=2,4,6}^{n-1}\left(\boldsymbol{X}_{i}-\boldsymbol{X}_{i-1}\right)\left(\boldsymbol{X}_{i}-\boldsymbol{X}_{i-1}\right)^{\prime}
$$

and

$$
\begin{gathered}
V_{\mathrm{MSSD}, n}= \\
\Phi^{-1}\left\{F_{p, \frac{1}{2}(n-2 p+1)}\left[\frac{(n-2 p+1)(n-1)}{2 n p}\right] T_{\mathrm{MSSD}, n}^{2}\right\}, \\
n=2 p+1,2 p+3, \ldots
\end{gathered}
$$

For even numbered observations, i.e., $n$, is an even number,

$$
T_{M S S D, n}^{2}=\left(\boldsymbol{X}_{n}-\overline{\boldsymbol{X}}_{n-1}\right)^{\prime} \boldsymbol{S}_{\mathrm{MSSD}, n-2}^{-1}\left(\boldsymbol{X}_{n}-\overline{\boldsymbol{X}}_{n-1}\right)
$$

where

$$
S_{\mathrm{MSSD}, n-2}=\frac{1}{2} \sum_{i=2,4,6}^{n-2}\left(\boldsymbol{X}_{i}-\boldsymbol{X}_{i-1}\right)\left(\boldsymbol{X}_{i}-\boldsymbol{X}_{i-1}\right)^{\prime}
$$

and

$$
\begin{aligned}
& V_{\mathrm{MSSD}, n}= \\
& \Phi^{-1}\left\{F_{p, \frac{1}{2}(n-2 p)}\left[\frac{(n-2 p)(n-1)}{2 n p}\right] T_{\mathrm{MSSD}, n}^{2}\right\}, \\
& n=2 p+2,2 p+4, \ldots
\end{aligned}
$$

For the $V_{\text {MSSD }}$ statistics in eqs. (5a), (5b), (6a) and (6b) above, $p$ is the number of quality characteristics monitored simultaneously, hence $p \geq 2$.

Tests for Shifts in the Mean Vector $\mu$

Because all the $V_{\text {MSSD }}$ statistics are standard normal random variables, the following tests will be used in the detection of shifts in the mean vector. Given a sequence of $V_{\text {MSSD }}$ statistics, i.e., $V_{\mathrm{MSSD}, a+1}, V_{\mathrm{MSSD}, a+2}, \ldots, V_{\mathrm{MSSD}, m}, \ldots$, where $V_{\mathrm{MSSD}, a}$ represents the control chart statistic, $V_{\mathrm{MSSD}}$, at observation $a$, the tests are defined as follow: 
The 1-of-1 Test: When $V_{\mathrm{MSSD}, m}$ is plotted, the test signals a shift in $\mu$ if $V_{\mathrm{MSSD}, m}>$ $3 \sigma$, i.e., $V_{\mathrm{MSSD}, m}>3$.

The 3-of-3 Test: When $V_{\mathrm{MSSD}, m}$ is plotted, the test signals a shift in $\mu$ if $V_{\mathrm{MSSD}, m}$, $V_{\mathrm{MSSD}, m-1}$ and $V_{\mathrm{MSSD}, m-2}$ all exceed $1 \sigma$ (i.e., 1 ). This test requires the availability of three consecutive $V_{\text {MSSD }}$ statistics.

The 4-of-5 Test: When $V_{\mathrm{MSSD}, m}$ is plotted, the test signals a shift in $\mu$ if at least four of the five values $V_{\mathrm{MSSD}, m}, V_{\mathrm{MSSD}, m-1}, \ldots$, $V_{\text {MSSD }, m-4}$ exceed $1 \sigma$ (i.e., 1 ). This test can only be used if five consecutive $V_{\text {MSSD }}$ statistics are available.

In addition to these tests, the EWMA chart computed from a sequence of the $V_{\text {MSSD }}$ statistics is also considered. The EWMA chart is defined as follows:

$$
\begin{aligned}
& Z_{\mathrm{MSSD}, m}=\alpha V_{\mathrm{MSSD}, m}+(1-\alpha) Z_{\mathrm{MSSD}, m-1}, \\
& m=a, a+1, \ldots
\end{aligned}
$$

where $Z_{\mathrm{MSSD}, a-1}=0$ and $a$ is an integer representing the starting point of the monitoring of a process. The $U C L$ of an EWMA chart is $K \sqrt{\alpha /(2-\alpha)}$, where $\alpha$ is the smoothing constant and $K$ is the control limit constant. For the simulation study in this paper, the values of $(\alpha, K)$ used are $(0.25,2.90)$ which gives $U C L=$ 1.096, i.e., similar to that in Ref. 1 .

Evaluating the Performance of the Enhanced Short Run Multivariate Chart

A simulation study is performed using SAS version 8 to study the performance of the enhanced short run multivariate chart for individual measurements. To enable a comparison to be made between the performance of the new short run chart with the chart proposed in Ref. 1, the simulation study of the new bivariate chart is conducted under the same condition as that of Ref. 1. The on-target mean vector vector is $\mu_{0}=(0,0)^{\prime}$ while the incontrol covariance matrix is $\Sigma_{0}=\left(\begin{array}{ll}1 & \rho \\ \rho & 1\end{array}\right)$ where $\rho$ is the correlation coefficient between the two quality characteristics. For every value of $c \in$ $\{10,20,50\}, c$ in-control observations are generated from a $N_{2}\left(\mu_{0}, \Sigma_{0}\right)$ distribution followed by 30 additional observations from a $N_{2}\left(\mu_{s}, \Sigma_{0}\right)$ distribution. The $V_{\text {MSSD }}$ statistics for cases KU and UU in Eq. (5a), (5b), (6a) and (6b) are computed as soon as enough values are available to define its statistics for the particular case.

This procedure is repeated 5000 times and the proportion of times an o.o.c. signal is observed from $c+1$ to $c+30$ for the first time is recorded. All of the tests defined in the previous section are used in evaluating the performance of the chart. Note that the new chart is also directionally invariant. Thus, the chart's performance is determined solely by the square root of the noncentrality parameter (see Ref. 1). Because of the directionally invariant property of the new short run multivariate chart, only $\mu_{s}$ $=(\delta, 0)^{\prime}$ based on $\rho=0$ and 0.5 are considered in the simulation study.

The results of cases KU and UU for the enhanced short run multivariate chart are given in Tables 2 and 3 for $\rho=0$ and 0.5 respectively. Tables 4 and 5 give the corresponding results of the short run multivariate chart proposed in Ref. 1. The results show that the approach incorporating the new estimator of process dispersion, i.e., $\boldsymbol{S}_{\mathrm{MSSD}}$, are superior to that proposed in Ref. 1.

For example, if $\delta=1.5, c=10$ and $\rho=$ 0 , the probabilities of detecting an o.o.c. for case KU are $0.225,0.721,0.681$ and 0.739 for the enhanced chart based on the 1-of-1, 3-of-3, 4-of5 and EWMA tests respectively (see Table 2). From the results in Table 4, the corresponding probabilities that are computed for these four tests are $0.056,0.253,0.172$ and 0.157 respectively. Clearly, these probabilities are much lower than those of the enhanced chart. Note also that the Type-I error of the enhanced chart based on the 3-of-3, 4-of-5 and EWMA tests are higher than those in Ref. 1. However, from Tables 2 and 3, it is observed that the probabilities of signaling a false o.o.c. for these three tests decrease as the values of $c$ increase. The probabilities of a false alarm for the 1-of-1 
Table 2. Simulation Results of the Enhanced Short Run Multivariate Chart for Cases KU and UU based on $\mu_{0}=(0,0)^{\prime}, \mu_{s}=(\delta, 0)^{\prime}$ and $\rho=0$.

\begin{tabular}{|c|c|c|c|c|c|c|c|c|c|c|c|c|c|}
\hline \multirow{2}{*}{\multicolumn{2}{|c|}{$\begin{array}{c}\rho=0 \\
\mu_{s}=(\delta, 0)^{\prime}\end{array}$}} & \multicolumn{4}{|c|}{$c=10$} & \multicolumn{4}{|c|}{$c=20$} & \multicolumn{4}{|c|}{$c=50$} \\
\hline & & 1-of-1 & 3 -of-3 & 4-of-5 & EWMA & 1-of-1 & 3 -of-3 & 4-of-5 & EWMA & 1-of-1 & 3 -of-3 & 4-of-5 & EWMA \\
\hline \multicolumn{14}{|l|}{$\delta$} \\
\hline \multirow[t]{2}{*}{0.0} & KU & 0.039 & 0.152 & 0.111 & 0.116 & 0.032 & 0.130 & 0.086 & 0.087 & 0.040 & 0.113 & 0.066 & 0.064 \\
\hline & UU & 0.036 & 0.156 & 0.111 & 0.118 & 0.035 & 0.123 & 0.079 & 0.087 & 0.037 & 0.113 & 0.063 & 0.069 \\
\hline \multirow[t]{2}{*}{0.5} & KU & 0.055 & 0.220 & 0.169 & 0.173 & 0.049 & 0.194 & 0.130 & 0.140 & 0.070 & 0.187 & 0.121 & 0.126 \\
\hline & UU & 0.040 & 0.171 & 0.126 & 0.133 & 0.037 & 0.149 & 0.100 & 0.108 & 0.054 & 0.158 & 0.096 & 0.102 \\
\hline \multirow[t]{2}{*}{1.0} & KU & 0.111 & 0.423 & 0.360 & 0.394 & 0.123 & 0.422 & 0.343 & 0.396 & 0.167 & 0.440 & 0.352 & 0.420 \\
\hline & UU & 0.049 & 0.221 & 0.168 & 0.174 & 0.063 & 0.239 & 0.171 & 0.189 & 0.114 & 0.305 & 0.228 & 0.266 \\
\hline \multirow[t]{2}{*}{1.5} & KU & & & & & & & & & & & & 0.846 \\
\hline & UU & 0.064 & 0.308 & 0.247 & 0.261 & 0.112 & 0.396 & 0.329 & 0.362 & 0.240 & 0.578 & 0.505 & 0.594 \\
\hline \multirow[t]{2}{*}{2.0} & KU & 0.409 & 0.919 & 0.910 & 0.947 & 0.510 & 0.943 & 0.931 & 0.970 & 0.665 & 0.972 & 0.968 & 0.991 \\
\hline & UU & 0.091 & 0.434 & 0.371 & 0.387 & 0.189 & 0.611 & 0.550 & 0.609 & 0.431 & 0.841 & 0.813 & 0.893 \\
\hline \multirow[t]{2}{*}{2.5} & KU & 0.611 & 0.986 & 0.986 & 1.000 & 0.740 & 0.994 & 0.994 & 0.998 & 0.882 & 0.999 & 0.998 & 1.000 \\
\hline & UU & 0.126 & 0.574 & 0.516 & 0.534 & 0.293 & 0.799 & 0.769 & 0.815 & 0.660 & 0.969 & 0.968 & 0.989 \\
\hline \multirow[t]{2}{*}{3.0} & KU & 0.787 & 0.998 & 0.999 & 1.000 & & 1.000 & 0.999 & & & 1.000 & 1.000 & 1.000 \\
\hline & UU & 0.173 & 0.718 & 0.678 & 0.681 & 0.430 & 0.927 & 0.914 & 0.939 & 0.849 & 0.997 & 0.998 & 1.000 \\
\hline \multirow[t]{2}{*}{4.0} & KU & 0.965 & 1.000 & 1.000 & 1.000 & 0.992 & 1.000 & 1.000 & 1.000 & 1.000 & 1.000 & 1.000 & 1.000 \\
\hline & UU & 0.292 & 0.910 & 0.897 & 0.897 & 0.695 & 0.996 & 0.995 & 0.998 & 0.988 & 1.000 & 1.000 & 1.000 \\
\hline \multirow[t]{2}{*}{5.0} & KU & 0.998 & 1.000 & 1.000 & 1.000 & 1.000 & 1.000 & 1.000 & 1.000 & 1.000 & 1.000 & 1.000 & 1.000 \\
\hline & UU & 0.423 & 0.980 & 0.981 & 0.978 & 0.883 & 1.000 & 1.000 & 1.000 & 1.000 & 1.000 & 1.000 & 1.000 \\
\hline
\end{tabular}


Table 3. Simulation Results of the Enhanced Short Run Multivariate Chart for Cases KU and UU based on $\mu_{0}=(0,0)^{\prime}, \mu_{s}=(\delta, 0)^{\prime}$ and $\rho=0.5$.

\begin{tabular}{|c|c|c|c|c|c|c|c|c|c|c|c|c|c|}
\hline \multirow{2}{*}{\multicolumn{2}{|c|}{$\begin{array}{c}\rho=0 \\
\mu_{s}=(\delta, 0)^{\prime}\end{array}$}} & \multicolumn{4}{|c|}{$c=10$} & \multicolumn{4}{|c|}{$c=20$} & \multicolumn{4}{|c|}{$c=50$} \\
\hline & & 1-of-1 & 3 -of-3 & 4-of-5 & EWMA & 1-of-1 & 3 -of-3 & $4-o f-5$ & EWMA & 1-of-1 & $3-$ of-3 & $4-$ of-5 & EWMA \\
\hline \multicolumn{14}{|c|}{$\delta$} \\
\hline \multirow[t]{2}{*}{0.0} & KU & 0.039 & 0.152 & 0.111 & 0.116 & 0.032 & 0.130 & 0.086 & 0.087 & 0.040 & 0.113 & 0.066 & 0.064 \\
\hline & UU & 0.036 & 0.156 & 0.111 & 0.118 & 0.035 & 0.123 & 0.079 & 0.087 & 0.037 & 0.113 & 0.063 & 0.069 \\
\hline \multirow[t]{2}{*}{0.5} & KU & 0.063 & 0.238 & 0.189 & 0.191 & 0.058 & 0.214 & 0.149 & 0.166 & 0.078 & 0.210 & 0.138 & 0.157 \\
\hline & UU & 0.040 & 0.179 & 0.132 & 0.137 & 0.040 & 0.163 & 0.113 & 0.118 & 0.061 & 0.171 & 0.108 & 0.120 \\
\hline \multirow[t]{2}{*}{1.0} & KU & 0.141 & 0.513 & 0.459 & 0.499 & 0.164 & 0.520 & 0.447 & 0.519 & 0.227 & 0.546 & 0.462 & 0.571 \\
\hline & UU & 0.055 & 0.245 & 0.191 & 0.198 & 0.076 & 0.281 & 0.216 & 0.237 & 0.148 & 0.387 & 0.295 & 0.359 \\
\hline \multirow[t]{2}{*}{1.5} & KU & 0.304 & 0.826 & 0.805 & 0.859 & 0.382 & 0.863 & 0.842 & 0.901 & 0.518 & 0.900 & 0.885 & 0.949 \\
\hline & UU & 0.078 & 0.364 & 0.307 & 0.317 & 0.144 & 0.498 & 0.429 & 0.484 & 0.322 & 0.709 & 0.662 & 0.760 \\
\hline \multirow[t]{2}{*}{2.0} & KU & 0.525 & 0.971 & 0.968 & 0.988 & 0.648 & 0.988 & 0.985 & 0.995 & 0.821 & 0.995 & 0.996 & 0.999 \\
\hline & UU & 0.112 & 0.522 & 0.466 & 0.478 & 0.255 & 0.734 & 0.692 & 0.744 & 0.572 & 0.932 & 0.923 & 0.973 \\
\hline \multirow[t]{2}{*}{2.5} & $\mathrm{KU}$ & 0.750 & 0.998 & 0.997 & 1.000 & 0.864 & 0.999 & 0.999 & 1.000 & 0.961 & 1.000 & 1.000 & 1.000 \\
\hline & UU & 0.157 & 0.679 & 0.639 & 0.645 & 0.404 & 0.900 & 0.888 & 0.924 & 0.810 & 0.994 & 0.995 & 0.999 \\
\hline \multirow[t]{2}{*}{3.0} & $\mathrm{KU}$ & 0.894 & 1.000 & 1.000 & 1.000 & 0.965 & 1.000 & 1.000 & 1.000 & 0.997 & 1.000 & 1.000 & 1.000 \\
\hline & UU & 0.217 & 0.822 & 0.796 & 0.793 & 0.556 & 0.976 & 0.974 & 0.985 & 0.941 & 1.000 & 1.000 & 1.000 \\
\hline \multirow[t]{2}{*}{4.0} & $\mathrm{KU}$ & 0.994 & 1.000 & 1.000 & 1.000 & 0.999 & 1.000 & 1.000 & 1.000 & 1.000 & 1.000 & 1.000 & 1.000 \\
\hline & UU & 0.371 & 0.962 & 0.958 & 0.959 & 0.826 & 1.000 & 1.000 & 1.000 & 0.999 & 1.000 & 1.000 & 1.000 \\
\hline \multirow[t]{2}{*}{5.0} & $\mathrm{KU}$ & 1.000 & 1.000 & 1.000 & 1.000 & 1.000 & 1.000 & 1.000 & 1.000 & 1.000 & 1.000 & 1.000 & 1.000 \\
\hline & UU & 0.537 & 0.996 & 0.995 & 0.996 & 0.959 & 1.000 & 1.000 & 1.000 & 1.000 & 1.000 & 1.000 & 1.000 \\
\hline
\end{tabular}


Table 4. Simulation Results of the Short Run Multivariate Chart in Ref. 1 for Cases KU and UU based on $\mu_{0}=(0,0)^{\prime}, \mu_{s}=(\delta, 0)^{\prime}$ and $\rho=0$.

\begin{tabular}{|c|c|c|c|c|c|c|c|c|c|c|c|c|c|}
\hline \multirow{2}{*}{\multicolumn{2}{|c|}{$\begin{array}{c}\rho=0 \\
\mu_{s}=(\delta, 0)^{\prime}\end{array}$}} & \multicolumn{4}{|c|}{$c=10$} & \multicolumn{4}{|c|}{$c=20$} & \multicolumn{4}{|c|}{$c=50$} \\
\hline & & 1-of-1 & 3-of-3 & 4-of-5 & EWMA & 1-of-1 & 3-of-3 & 4-of-5 & EWMA & 1-of-1 & $3-o f-3$ & 4-of-5 & EWMA \\
\hline \multicolumn{14}{|c|}{$\delta$} \\
\hline \multirow[t]{2}{*}{0.0} & KU & 0.041 & 0.102 & 0.052 & 0.038 & 0.037 & 0.103 & 0.046 & 0.044 & 0.042 & 0.103 & 0.056 & 0.042 \\
\hline & UU & 0.040 & 0.103 & 0.052 & 0.039 & 0.039 & 0.100 & 0.049 & 0.041 & 0.038 & 0.101 & 0.050 & 0.043 \\
\hline \multirow[t]{2}{*}{0.5} & KU & 0.048 & 0.120 & 0.069 & 0.056 & 0.049 & 0.133 & 0.073 & 0.066 & 0.057 & 0.153 & 0.088 & 0.088 \\
\hline & UU & 0.041 & 0.100 & 0.054 & 0.040 & 0.040 & 0.106 & 0.053 & 0.049 & 0.051 & 0.131 & 0.070 & 0.069 \\
\hline \multirow[t]{2}{*}{1.0} & KU & 0.052 & 0.178 & 0.110 & 0.093 & 0.072 & 0.233 & 0.149 & 0.151 & 0.113 & 0.312 & 0.221 & 0.263 \\
\hline & UU & 0.043 & 0.112 & 0.062 & 0.051 & 0.052 & 0.143 & 0.084 & 0.080 & 0.087 & 0.225 & 0.154 & 0.167 \\
\hline \multirow[t]{2}{*}{1.5} & $\mathrm{KU}$ & 0.056 & 0.253 & 0.172 & 0.157 & 0.093 & 0.387 & 0.286 & 0.321 & 0.184 & 0.581 & 0.493 & 0.617 \\
\hline & UU & 0.041 & 0.128 & 0.074 & 0.065 & 0.067 & 0.216 & 0.141 & 0.148 & 0.144 & 0.417 & 0.320 & 0.393 \\
\hline \multirow[t]{2}{*}{2.0} & KU & 0.069 & 0.340 & 0.248 & 0.247 & 0.132 & 0.558 & 0.469 & 0.536 & 0.292 & 0.821 & 0.785 & 0.903 \\
\hline & UU & 0.049 & 0.164 & 0.104 & 0.091 & 0.096 & 0.329 & 0.241 & 0.270 & 0.233 & 0.652 & 0.585 & 0.713 \\
\hline \multirow[t]{2}{*}{2.5} & KU & 0.096 & 0.434 & 0.337 & 0.342 & 0.193 & 0.713 & 0.650 & 0.741 & 0.445 & 0.949 & 0.943 & 0.991 \\
\hline & UU & 0.064 & 0.215 & 0.145 & 0.133 & 0.151 & 0.468 & 0.381 & 0.428 & 0.368 & 0.841 & 0.809 & 0.921 \\
\hline \multirow[t]{2}{*}{3.0} & KU & 0.131 & 0.522 & 0.425 & 0.442 & 0.290 & 0.833 & 0.789 & 0.882 & 0.617 & 0.991 & 0.991 & 1.000 \\
\hline & UU & 0.096 & 0.269 & 0.184 & 0.181 & 0.232 & 0.611 & 0.528 & 0.603 & 0.539 & 0.947 & 0.942 & 0.991 \\
\hline \multirow[t]{2}{*}{4.0} & KU & 0.268 & 0.663 & 0.561 & 0.605 & 0.569 & 0.949 & 0.933 & 0.984 & 0.914 & 1.000 & 1.000 & 1.000 \\
\hline & UU & 0.194 & 0.372 & 0.258 & 0.292 & 0.484 & 0.804 & 0.733 & 0.854 & 0.873 & 0.996 & 0.997 & 1.000 \\
\hline \multirow[t]{2}{*}{5.0} & KU & 0.473 & 0.747 & 0.652 & 0.730 & 0.832 & 0.984 & 0.980 & 0.999 & 0.996 & 1.000 & 1.000 & 1.000 \\
\hline & UU & 0.355 & 0.448 & 0.304 & 0.397 & 0.769 & 0.900 & 0.851 & 0.957 & 0.987 & 1.000 & 1.000 & 1.000 \\
\hline
\end{tabular}


Table 5. Simulation Results of the Short Run Multivariate Chart in Ref. 1 for Cases KU and UU based on $\mu_{0}=(0,0)^{\prime}, \mu_{s}=(\delta, 0)^{\prime}$ and $\rho=0.5$.

\begin{tabular}{|c|c|c|c|c|c|c|c|c|c|c|c|c|c|}
\hline \multirow{2}{*}{\multicolumn{2}{|c|}{$\begin{array}{c}\rho=0 \\
\mu_{s}=(\delta, 0)^{\prime}\end{array}$}} & \multicolumn{4}{|c|}{$c=10$} & \multicolumn{4}{|c|}{$c=20$} & \multicolumn{4}{|c|}{$c=50$} \\
\hline & & 1-of-1 & 3 -of-3 & 4-of-5 & EWMA & 1-of-1 & 3 -of-3 & $4-$ of-5 & EWMA & 1-of-1 & 3 -of-3 & 4-of-5 & EWMA \\
\hline \multicolumn{14}{|c|}{$\delta$} \\
\hline \multirow[t]{2}{*}{0.0} & KU & 0.041 & 0.102 & 0.052 & 0.038 & 0.037 & 0.103 & 0.046 & 0.044 & 0.042 & 0.103 & 0.056 & 0.042 \\
\hline & UU & 0.040 & 0.103 & 0.052 & 0.039 & 0.039 & 0.100 & 0.049 & 0.041 & 0.038 & 0.101 & 0.050 & 0.043 \\
\hline \multirow[t]{2}{*}{0.5} & KU & 0.047 & 0.124 & 0.072 & 0.059 & 0.052 & 0.141 & 0.082 & 0.082 & 0.065 & 0.166 & 0.101 & 0.102 \\
\hline & UU & 0.042 & 0.102 & 0.055 & 0.041 & 0.041 & 0.115 & 0.063 & 0.049 & 0.054 & 0.144 & 0.079 & 0.079 \\
\hline \multirow[t]{2}{*}{1.0} & KU & 0.054 & 0.196 & 0.124 & 0.120 & 0.077 & 0.274 & 0.190 & 0.201 & 0.129 & 0.391 & 0.295 & 0.355 \\
\hline & UU & 0.042 & 0.115 & 0.068 & 0.050 & 0.056 & 0.165 & 0.098 & 0.097 & 0.098 & 0.281 & 0.197 & 0.217 \\
\hline \multirow[t]{2}{*}{1.5} & KU & 0.061 & 0.286 & 0.202 & 0.199 & 0.109 & 0.465 & 0.374 & 0.428 & 0.234 & 0.700 & 0.638 & 0.789 \\
\hline & UU & 0.047 & 0.139 & 0.087 & 0.077 & 0.079 & 0.266 & 0.181 & 0.199 & 0.181 & 0.527 & 0.440 & 0.553 \\
\hline \multirow[t]{2}{*}{2.0} & KU & 0.085 & 0.399 & 0.308 & 0.305 & 0.171 & 0.650 & 0.588 & 0.679 & 0.387 & 0.916 & 0.903 & 0.976 \\
\hline & UU & 0.062 & 0.182 & 0.119 & 0.121 & 0.126 & 0.416 & 0.325 & 0.364 & 0.314 & 0.785 & 0.744 & 0.870 \\
\hline \multirow[t]{2}{*}{2.5} & KU & 0.127 & 0.501 & 0.402 & 0.421 & 0.269 & 0.804 & 0.769 & 0.857 & 0.589 & 0.984 & 0.985 & 0.999 \\
\hline & UU & 0.091 & 0.250 & 0.167 & 0.173 & 0.218 & 0.578 & 0.495 & 0.564 & 0.508 & 0.927 & 0.922 & 0.983 \\
\hline \multirow[t]{2}{*}{3.0} & KU & 0.187 & 0.590 & 0.490 & 0.527 & 0.418 & 0.900 & 0.884 & 0.951 & 0.789 & 0.998 & 0.998 & 1.000 \\
\hline & UU & 0.139 & 0.317 & 0.217 & 0.229 & 0.341 & 0.717 & 0.645 & 0.733 & 0.719 & 0.979 & 0.983 & 0.999 \\
\hline \multirow[t]{2}{*}{4.0} & KU & 0.394 & 0.724 & 0.626 & 0.686 & 0.751 & 0.977 & 0.970 & 0.996 & 0.981 & 1.000 & 1.000 & 1.000 \\
\hline & UU & 0.293 & 0.424 & 0.288 & 0.354 & 0.678 & 0.883 & 0.831 & 0.935 & 0.965 & 1.000 & 1.000 & 1.000 \\
\hline \multirow[t]{2}{*}{5.0} & KU & 0.653 & 0.801 & 0.700 & 0.802 & 0.944 & 0.995 & 0.993 & 1.000 & 1.000 & 1.000 & 1.000 & 1.000 \\
\hline & UU & 0.518 & 0.489 & 0.325 & 0.473 & 0.909 & 0.949 & 0.911 & 0.989 & 0.999 & 1.000 & 1.000 & 1.000 \\
\hline
\end{tabular}


Table 6. $V_{\mathrm{MSSD}}$ and $V$ Statistics for Case UU.

\begin{tabular}{|c|cccc|c|cccc|}
\hline $\begin{array}{c}\text { Observation } \\
\text { No., } n\end{array}$ & $X_{1}$ & $X_{2}$ & $V_{n}$ & $V_{\text {MSSD }, n}$ & $\begin{array}{c}\text { Observation } \\
\text { No., } n\end{array}$ & $X_{1}$ & $X_{2}$ & $V_{n}$ & $V_{\text {MSSD }, n}$ \\
\hline 1 & 1.404 & 0.268 & - & - & 21 & 0.819 & -0.277 & 0.395 & 0.580 \\
2 & 0.624 & 1.392 & - & - & 22 & 1.706 & 0.564 & 0.780 & 1.085 \\
3 & 0.454 & 0.755 & - & - & 23 & 1.198 & -1.313 & 2.181 & 2.434 \\
4 & -1.768 & -1.902 & 1.162 & - & 24 & 2.863 & 0.211 & 2.049 & 2.737 \\
5 & -0.224 & 0.140 & -1.452 & -1.650 & 25 & 2.141 & 0.438 & 0.545 & 1.657 \\
6 & -0.082 & 0.734 & -0.585 & -1.214 & 26 & 1.823 & 0.474 & -0.023 & 0.987 \\
7 & 1.146 & 0.484 & -0.190 & -0.327 & 27 & 1.609 & 0.414 & -0.366 & 0.630 \\
8 & 1.816 & 0.906 & 0.222 & 0.058 & 28 & 2.811 & 2.192 & 1.191 & 1.650 \\
9 & -1.245 & -1.555 & 0.482 & 0.296 & 29 & 0.170 & -0.650 & -0.987 & -0.676 \\
10 & -0.976 & -0.340 & -0.199 & 0.023 & 30 & -0.776 & -1.186 & -0.193 & 0.347 \\
11 & -0.621 & -1.058 & -0.266 & -0.393 & 31 & -0.111 & -0.613 & -1.216 & -0.838 \\
12 & -0.080 & -0.710 & -0.507 & -0.800 & 32 & 1.400 & 0.302 & -0.656 & 0.313 \\
13 & 0.742 & -0.146 & -0.202 & 0.042 & 33 & 1.584 & 0.337 & -0.403 & 0.609 \\
14 & -0.543 & -0.818 & -0.824 & -0.654 & 34 & 2.047 & 0.585 & 0.080 & 1.203 \\
15 & -2.335 & -2.801 & 1.437 & 1.507 & 35 & 0.481 & 0.690 & -0.153 & 0.667 \\
16 & -0.848 & -1.176 & -0.808 & -0.415 & 36 & 3.773 & 2.495 & 1.693 & 2.545 \\
17 & -0.431 & 0.590 & 0.836 & 0.742 & 37 & 1.891 & 1.871 & 0.673 & 1.256 \\
18 & 1.369 & 1.863 & 0.769 & 0.955 & 38 & 2.169 & 1.073 & -0.160 & 0.420 \\
19 & 0.283 & 0.197 & -1.659 & -1.405 & 39 & 1.761 & 1.191 & -0.400 & 0.049 \\
20 & 0.850 & 0.149 & -0.155 & 0.028 & 40 & 1.184 & -0.113 & -0.531 & 0.132 \\
\hline
\end{tabular}

Figure 1. Plotted $V_{\text {MSSD }}$ Statistics for Case UU

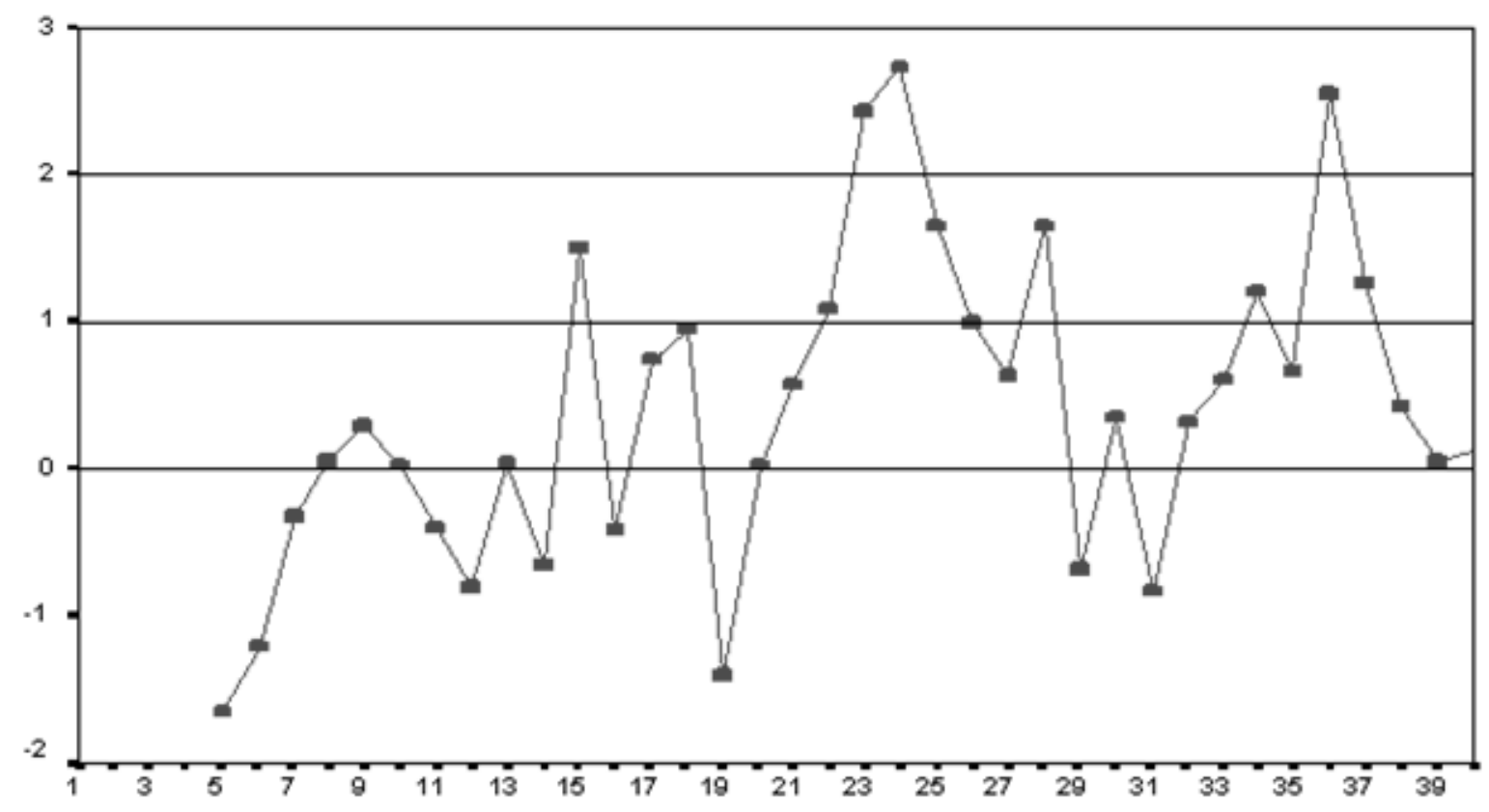

Observation Number 
Figure 2. Plotted $V$ Statistics for Case UU

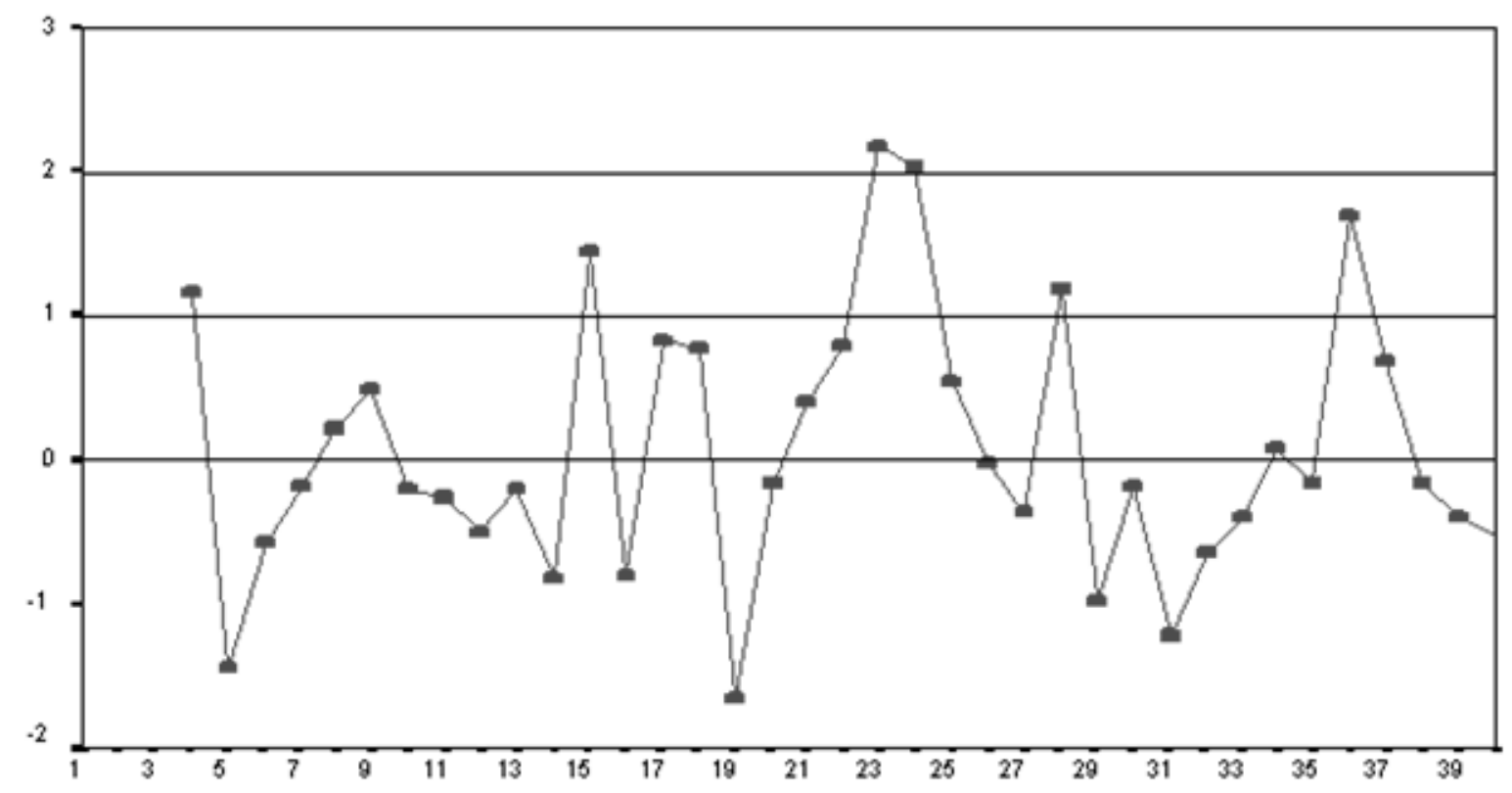

Observation Number

test in Tables 2, 3, 4 and 5 are almost the same. The results also show that the performance of the enhanced chart based on the basic 1-of-1 rule is superior to the chart proposed in Ref. 1 .

\section{An Example of Application}

An example will be given to show how the proposed enhanced short run multivariate chart is put to work. To simulate an in-control process, 20 bivariate observations are generated using SAS version 8 from a $N_{2}\left(\mu_{0}, \Sigma_{0}\right)$ distribution. For an o.o.c. process, with a shift in the mean vector, the next 20 bivariate observations are generated from a $N_{2}\left(\mu_{s}, \Sigma_{0}\right)$ distribution. Here, $\mu_{0}=\left(\begin{array}{l}0 \\ 0\end{array}\right), \quad \mu_{s}=\left(\begin{array}{c}1.3 \\ 0\end{array}\right)$, $\Sigma_{0}=\left(\begin{array}{ll}1 & \rho \\ \rho & 1\end{array}\right)$ where $\rho=0.8$. The 40 observations generated are substituted in eqs. (6a) and (6b) to compute the corresponding $V_{\text {MSSD }}$ statistics for case UU. Similarly, these 40 observations are substituted in Eq. (4) to compute the corresponding $V$ statistics for case UU. The computed $V$ and $V_{\text {MSSD }}$ statistics are summarized in Table 6. Figures 1 and 2 show the plotted $V_{\text {MSSD }}$ and $V$ statistics respectively. For the enhanced chart based on the $V_{\text {MSSD }}$ statistics, the 3-of-3 test signals an o.o.c. at observation 24 while the 4 -of-5 test signals at observation 25. The chart proposed in Ref. 1 based on the $V$ statistics fails to detect a shift in the mean vector.

\section{Conclusion}

It is shown in this paper that the enhanced chart based on a robust estimator of scale, i.e., $\boldsymbol{S}_{\text {MSSD }}$ gives excellent improvement over the existing short run multivariate chart proposed in Khoo \& Quah (2002). The proofs of how the $V_{\text {MSsD }}$ statistics for cases KU and UU are derived are shown in the Appendix. 


\section{References}

Khoo, M. B. C. \& Quah, S. H. (2002). Proposed short runs multivariate control charts for the process mean. Quality Engineering, 14 (4), $603-621$.

Holmes, D. S., \& Mergen, A. E. (1993). Improving the performance of the $T^{2}$ control chart. Quality Engineering, 5 (4), 619 - 625.

Seber, G. A. F. (1984). Multivariate observations. New York : John Wiley and Sons.

\section{Appendix}

In this section, it will be shown that the $V_{\text {MSSD }}$ statistics in eqs. (5a), (5b), (6a) and (6b) are $N(0,1)$ random variables. All the notations used here are already defined in the earlier sections. The following theorems taken from Seber (1984) are used:

Theorem A. Suppose that $y \sim N_{p}(\mathbf{0}, \Sigma)$, $W \sim W_{p}(n, \Sigma)$, and $y$ and $W$ are statistically independent. Assumed that the distribution are nonsingular, i.e., $\Sigma>\mathbf{O}$, and $n \geq p$, so that $\boldsymbol{W}^{-1}$ exists with probability 1 .

Let

$$
T^{2}=n y^{\prime} \boldsymbol{W}^{-1} y^{\prime},
$$

then

$$
\frac{(n-p+1)}{p} \frac{T^{2}}{n} \sim F_{p, n-p+1}
$$

Theorem B. Suppose that $\boldsymbol{X}_{1}, \boldsymbol{X}_{2}, \ldots, \boldsymbol{X}_{n}$ are independently and identically distributed (i.i.d.) as $N_{p}(\mathbf{0}, \Sigma)$, then

$$
\sum_{i=1}^{n} \boldsymbol{X}_{i} \boldsymbol{X}_{i}^{\prime} \sim W_{p}(n, \Sigma)
$$

where $W_{p}(n, \Sigma)$ is the Wishart distribution with $n$ degrees of freedom.

\section{Equation (5a): Case KU}

We need to show that for odd numbered observations, i.e., when $n$ is an odd number,

$$
T_{\mathrm{MSSD}, n}^{2}=\left(\boldsymbol{X}_{n}-\mu_{0}\right)^{\prime} \boldsymbol{S}_{\mathrm{MSSD}, n-1}^{-1}\left(\boldsymbol{X}_{n}-\mu_{0}\right)
$$

$$
\sim \frac{2 p}{n-2 p+1} F_{p, \frac{1}{2}(n-2 p+1)}
$$

Proof:

If $\boldsymbol{X}_{j}, j=1,2,3, \ldots$, are i.i.d. $N_{p}(\mu, \Sigma)$

variables, then

$$
\boldsymbol{X}_{i}-\boldsymbol{X}_{i-1} \sim N_{p}(\mathbf{0}, 2 \Sigma), i=2,4,6, \ldots
$$

and

$$
\frac{1}{\sqrt{2}}\left(\boldsymbol{X}_{i}-\boldsymbol{X}_{i-1}\right) \sim N_{p}(\mathbf{0}, \Sigma), i=2,4,6, \ldots
$$

Thus, from eq. (A3) of Theorem B,

$\frac{1}{2} \sum_{i=2,4,6}^{n-1}\left(\boldsymbol{X}_{i}-\boldsymbol{X}_{i-1}\right)\left(\boldsymbol{X}_{i}-\boldsymbol{X}_{i-1}\right)^{\prime} \sim W_{p}\left(\frac{n-1}{2}, \Sigma\right)$,

i.e.,

$$
S_{\mathrm{MSSD}, n-1} \sim W_{p}\left(\frac{n-1}{2}, \Sigma\right) .
$$

Because $\mu=\mu_{0}$ is known, then

$$
\boldsymbol{X}_{n}-\mu_{0} \sim N_{p}(\mathbf{0}, \Sigma)
$$

Substituting Eq. (A4) and (A5) into Eq. (A1) and (A2) of Theorem A,

$$
\begin{aligned}
& \frac{\left(\frac{n-1}{2}-p+1\right)\left(\frac{n-1}{2}\right)}{p\left(\frac{n-1}{2}\right)}\left(\boldsymbol{X}_{n}-\mu_{0}\right)^{\prime} \boldsymbol{S}_{\mathrm{MSSD}, n-1}^{-1}\left(\boldsymbol{X}_{n}-\boldsymbol{\mu}_{0}\right) \\
& \sim F_{p, \frac{n-1}{2}-p+1} \\
& \text { i.e., } \\
& \frac{(n-2 p+1)}{2 p}\left(\boldsymbol{X}_{n}-\mu_{0}\right)^{\prime} \boldsymbol{S}_{\mathrm{MSSD}, n-1}^{-1}\left(\boldsymbol{X}_{n}-\boldsymbol{\mu}_{0}\right) \\
& \sim F_{p, \frac{1}{2}(n-2 p+1)} .
\end{aligned}
$$

Define

$$
T_{\mathrm{MSSD}, n}^{2}=\left(\boldsymbol{X}_{n}-\mu_{0}\right)^{\prime} S_{\mathrm{MSSD}, n-1}^{-1}\left(\boldsymbol{X}_{n}-\mu_{0}\right) ;
$$

then

$T_{\mathrm{MSSD}, n}^{2} \sim \frac{2 p}{n-2 p+1} F_{p, \frac{1}{2}(n-2 p+1)}$ for $n>2 p-1$, i.e., $n=2 p+1,2 p+3, \ldots$. 
Equation (5b): Case KU

We need to show that for even numbered observations, i.e., when $n$ is an even number,

$$
\begin{aligned}
& T_{\mathrm{MSSD}, n}^{2}=\left(\boldsymbol{X}_{n}-\mu_{0}\right)^{\prime} \boldsymbol{S}_{\mathrm{MSSD}, n-2}^{-1}\left(\boldsymbol{X}_{n}-\mu_{0}\right) \\
& \sim \frac{2 p}{n-2 p} F_{p, \frac{1}{2}(n-2 p)}
\end{aligned}
$$

Proof:

If $\boldsymbol{X}_{j}, j=1,2,3, \ldots$, are i.i.d. $N_{p}(\mu, \Sigma)$ variables, then

$$
\boldsymbol{X}_{i}-\boldsymbol{X}_{i-1} \sim N_{p}(\mathbf{0}, 2 \Sigma), i=2,4,6, \ldots
$$

and

$$
\frac{1}{\sqrt{2}}\left(\boldsymbol{X}_{i}-\boldsymbol{X}_{i-1}\right) \sim N_{p}(\mathbf{0}, \Sigma), i=2,4,6, \ldots .
$$

Thus, from Eq. (A3) of Theorem B,

$\frac{1}{2} \sum_{i=2,4,6}^{n-2}\left(\boldsymbol{X}_{i}-\boldsymbol{X}_{i-1}\right)\left(\boldsymbol{X}_{i}-\boldsymbol{X}_{i-1}\right)^{\prime} \sim W_{p}\left(\frac{n-2}{2}, \Sigma\right)$,

i.e.,

$$
S_{\mathrm{MSSD}, n-2} \sim W_{p}\left(\frac{n-2}{2}, \Sigma\right) .
$$

Because $\mu=\mu_{0}$ is known, then

$$
\boldsymbol{X}_{n}-\mu_{0} \sim N_{p}(\mathbf{0}, \Sigma)
$$

Substituting Eq. (A6) and (A7) into Eq. (A1) and (A2) of Theorem A,

$$
\begin{aligned}
& \frac{\left(\frac{n-2}{2}-p+1\right)\left(\frac{n-2}{2}\right)}{p\left(\frac{n-2}{2}\right)}\left(X_{n}-\mu_{0}\right)^{\prime} S_{\mathrm{MSSD}, n-2}^{-1}\left(X_{n}-\mu_{0}\right) \\
& \sim F_{p, \frac{n-2}{2}-p+1} \\
& \text { i.e., } \\
& \frac{(n-2 p)}{2 p}\left(\boldsymbol{X}_{n}-\mu_{0}\right)^{\prime} \boldsymbol{S}_{\mathrm{MSSD}, n-2}^{-1}\left(\boldsymbol{X}_{n}-\mu_{0}\right) \\
& \sim F_{p, \frac{1}{2}(n-2 p)} .
\end{aligned}
$$

Define

$$
T_{\mathrm{MSSD}, n}^{2}=\left(\boldsymbol{X}_{n}-\mu_{0}\right)^{\prime} S_{\mathrm{MSSD}, n-2}^{-1}\left(\boldsymbol{X}_{n}-\mu_{0}\right)
$$

then

$T_{\mathrm{MSSD}, n}^{2} \sim \frac{2 p}{n-2 p} F_{p, \frac{1}{2}(n-2 p)}$ for $n>2 p$,

i.e., $n=2 p+2,2 p+4, \ldots$.

Equation (6a): Case UU

We need to show that for odd numbered observations, i.e., when $n$ is an odd number,

$$
\begin{aligned}
& T_{\mathrm{MSSD}, n}^{2}=\left(\boldsymbol{X}_{n}-\overline{\boldsymbol{X}}_{n-1}\right)^{\prime} \boldsymbol{S}_{\mathrm{MSSD}, n-1}^{-1}\left(\boldsymbol{X}_{n}-\overline{\boldsymbol{X}}_{n-1}\right) \sim \\
& \frac{2 n p}{(n-2 p+1)(n-1)} F_{p, \frac{1}{2}(n-2 p+1)}
\end{aligned}
$$

Proof:

If $\boldsymbol{X}_{j}, j=1,2,3, \ldots$, are i.i.d. $N_{p}(\mu, \Sigma)$ variables, then

$$
\boldsymbol{X}_{i}-\boldsymbol{X}_{i-1} \sim N_{p}(\mathbf{0}, 2 \Sigma), i=2,4,6, \ldots
$$

and

$\frac{1}{\sqrt{2}}\left(\boldsymbol{X}_{i}-\boldsymbol{X}_{i-1}\right) \sim N_{p}(\mathbf{0}, \Sigma), i=2,4,6, \ldots$

Thus, from Eq. (A3) of Theorem B,

$\frac{1}{2} \sum_{i=2,4,6}^{n-1}\left(\boldsymbol{X}_{i}-\boldsymbol{X}_{i-1}\right)\left(\boldsymbol{X}_{i}-\boldsymbol{X}_{i-1}\right)^{\prime} \sim W_{p}\left(\frac{n-1}{2}, \Sigma\right)$,

i.e.,

$$
S_{\mathrm{MSSD}, n-1} \sim W_{p}\left(\frac{n-1}{2}, \Sigma\right) .
$$

Because $\mu$ is unknown,

$$
\overline{\boldsymbol{X}}_{n-1} \sim N_{p}\left(\mu, \frac{\Sigma}{n-1}\right)
$$

Then,

$$
\begin{aligned}
& \boldsymbol{X}_{n}-\overline{\boldsymbol{X}}_{n-1} \sim N_{p}\left[\mathbf{0},\left(1+\frac{1}{n-1}\right) \Sigma\right] \equiv \\
& N_{p}\left(\mathbf{0}, \frac{n}{n-1} \Sigma\right)
\end{aligned}
$$

and 


$$
\sqrt{\frac{n-1}{n}}\left(\boldsymbol{X}_{n}-\overline{\boldsymbol{X}}_{n-1}\right) \sim N_{p}(\mathbf{0}, \Sigma)
$$

Substituting Eq. (A8) and (A9) into Eq. (A1) and (A2) of Theorem A,

$$
\begin{aligned}
& \frac{\left(\frac{n-1}{2}-p+1\right)\left(\frac{n-1}{2}\right)\left(\frac{n-1}{n}\right)}{p\left(\frac{n-1}{2}\right)} \\
& \left(\boldsymbol{X}_{n}-\overline{\boldsymbol{X}}_{n-1}\right)^{\prime} \boldsymbol{S}_{\mathrm{MSSD}, n-1}^{-1}\left(\boldsymbol{X}_{n}-\overline{\boldsymbol{X}}_{n-1}\right) \sim F_{p, \frac{n-1}{2}-p+1}
\end{aligned}
$$

i.e.,

$$
\begin{gathered}
\frac{(n-2 p+1)(n-1)}{2 n p} \\
\left(\boldsymbol{X}_{n}-\overline{\boldsymbol{X}}_{n-1}\right)^{\prime} \boldsymbol{S}_{\mathrm{MSSD}, n-1}^{-1}\left(\boldsymbol{X}_{n}-\overline{\boldsymbol{X}}_{n-1}\right) \sim F_{p, \frac{1}{2}(n-2 p+1)}
\end{gathered}
$$

Define

$T_{\mathrm{MSSD}, n}^{2}=\left(\boldsymbol{X}_{n}-\overline{\boldsymbol{X}}_{n-1}\right)^{\prime} \boldsymbol{S}_{\mathrm{MSSD}, n-1}^{-1}\left(\boldsymbol{X}_{n}-\overline{\boldsymbol{X}}_{n-1}\right) ;$

then

$T_{\mathrm{MSSD}, n}^{2} \sim \frac{2 n p}{(n-2 p+1)(n-1)} F_{p, \frac{1}{2}(n-2 p+1)}$

for $n>2 p-1$, i.e., $n=2 p+1,2 p+3, \ldots$.

Equation (6b): Case UU

We need to show that for even numbered observations, i.e., when $n$ is an even number,

$T_{\mathrm{MSS}, n}^{2}=\left(\boldsymbol{X}_{n}-\overline{\boldsymbol{X}}_{n-1}\right)^{\prime} \boldsymbol{S}_{\mathrm{MSSD}, n-2}^{-1}\left(\boldsymbol{X}_{n}-\overline{\boldsymbol{X}}_{n-1}\right) \sim$ $\frac{2 n p}{(n-2 p)(n-1)} F_{p, \frac{1}{2}(n-2 p)}$

Proof:

If $\boldsymbol{X}_{j}, j=1,2,3, \ldots$, are i.i.d. $N_{p}(\mu, \Sigma)$ variables, then

$$
\boldsymbol{X}_{i}-\boldsymbol{X}_{i-1} \sim N_{p}(\mathbf{0}, 2 \Sigma), i=2,4,6, \ldots
$$

and

$$
\frac{1}{\sqrt{2}}\left(\boldsymbol{X}_{i}-\boldsymbol{X}_{i-1}\right) \sim N_{p}(\mathbf{0}, \Sigma), i=2,4,6, \ldots .
$$

Thus, from Eq. (A3) of Theorem B,

$$
\frac{1}{2} \sum_{i=2,4,6}^{n-2}\left(\boldsymbol{X}_{i}-\boldsymbol{X}_{i-1}\right)\left(\boldsymbol{X}_{i}-\boldsymbol{X}_{i-1}\right)^{\prime} \sim W_{p}\left(\frac{n-2}{2}, \Sigma\right),
$$

i.e.,

$$
S_{\mathrm{MSSD}, n-2} \sim W_{p}\left(\frac{n-2}{2}, \Sigma\right) .
$$

Because $\mu$ is unknown,

$$
\overline{\boldsymbol{X}}_{n-1} \sim N_{p}\left(\mu, \frac{\Sigma}{n-1}\right)
$$

Then,

$$
\boldsymbol{X}_{n}-\overline{\boldsymbol{X}}_{n-1} \sim N_{p}\left[\mathbf{0},\left(\frac{n}{n-1}\right) \Sigma\right]
$$

and

$$
\sqrt{\frac{n-1}{n}}\left(\boldsymbol{X}_{n}-\overline{\boldsymbol{X}}_{n-1}\right) \sim N_{p}(\mathbf{0}, \Sigma)
$$

Substituting Eq. (A10) and (A11) into Eq. (A1) and (A2) of Theorem A,

$$
\begin{aligned}
& \frac{\left(\frac{n-2}{2}-p+1\right)\left(\frac{n-2}{2}\right)\left(\frac{n-1}{n}\right)}{p\left(\frac{n-2}{2}\right)} \\
& \left(\boldsymbol{X}_{n}-\overline{\boldsymbol{X}}_{n-1}\right)^{\prime} \boldsymbol{S}_{\mathrm{MSSD}, n-2}^{-1}\left(\boldsymbol{X}_{n}-\overline{\boldsymbol{X}}_{n-1}\right) \sim F_{p, \frac{n-2}{2}-p+1}
\end{aligned}
$$

i.e.,

$$
\begin{gathered}
\frac{(n-2 p)(n-1)}{2 n p} \\
\left(\boldsymbol{X}_{n}-\overline{\boldsymbol{X}}_{n-1}\right)^{\prime} \boldsymbol{S}_{\mathrm{MSSD}, n-2}^{-1}\left(\boldsymbol{X}_{n}-\overline{\boldsymbol{X}}_{n-1}\right) \sim F_{p, \frac{n-2 p}{2}}
\end{gathered}
$$

Define

$$
T_{\mathrm{MSSD}, n}^{2}=\left(\boldsymbol{X}_{n}-\bar{X}_{n-1}\right)^{\prime} S_{\mathrm{MSSD}, n-2}^{-1}\left(X_{n}-\bar{X}_{n-1}\right) \text {; }
$$

then

$$
\begin{aligned}
& T_{\mathrm{MSSD}, n}^{2} \sim \frac{2 n p}{(n-2 p)(n-1)} F_{p, \frac{1}{2}(n-2 p)} \text { for } n>2 p, \\
& \text { i.e., } n=2 p+2,2 p+4, \ldots
\end{aligned}
$$

